\title{
No benefit from prophylactic antibiotics in third molar surgery
}

\author{
Does prophylactic antibiotic use reduce infection following third molar \\ removal?
}

Poeschl PW, Eckel D, Poeschl E. Postoperative prophylactic antibiotic treatment in third molar surgery - a necessity? J Oral Maxillofac Surg 2004; 62:3-8

Design This was a randomised controlled trial in a hospital environment.

Intervention After surgical removal of third molars postoperative treatment was with oral amoxicillin-clavulanic acid (AC) or clindamycin $(C L)$ or no antibiotics (C). The surgical technique was the same in all cases, and the follow-up period was 4 weeks.

Outcome measure Parameters evaluated were pain, differences in mouth opening, infection, the occurrence of dry socket, and adverse postoperative side effects.

Results A total of 528 lower third molars were surgically removed in 288 patients during a period of 30 months. The patients' mean age was 20.7 years (age range, 14-61 years). No severe complications such as perimandibular abscess or cellulitis occurred in any patient in any group. There was no significant difference between the groups in the overall occurrence of local infection symptoms after surgery (range, 3.4-4.4\%; mean, 3.98\%), nor for other parameters. Interestingly, $69.6 \%$ of the patients with dry sockets had partially erupted third molars. This rate was the same in each group $(62.5 \%$ versus $75 \%)$ and did not vary significantly. Reported adverse effects were similar in each group (15.3\% for AC, $12.2 \%$ for $C L, 13.9 \%$ for C).

Conclusions The results show that specific postoperative oral prophylactic antibiotic treatment after the removal of lower third molars does not contribute to better wound healing, less pain nor increased mouth opening, and could not prevent the cases of inflammatory problems after surgery, respectively. It is therefore not recommended for routine use.

\section{Commentary}

The use of prophylactic antibiotic therapy following third molar surgery is a common, if not universal, practice. There is very little evidence to support its routine use and there is, however, a growing body of data to suggest that this practice wastes resources with very little prospect of health gain. The use of broad-spectrum, thirdgeneration antibiotics seems completely unacceptable, as this study has also shown.

The authors present a randomised (but not blind or placebocontrolled) study of 288 patients, most of whom had two lower third molars removed. They were randomly allocated to receive oral amoxicillin with clavulanic acid or clindamycin. A third group received no treatment at all. Whereas the patients' mean age was 20.7 years, the range of $14-61$ years suggests that a large proportion were quite young. In addition, those who smoked were excluded from the study.

The analysis of the results is difficult to audit because there are four factors listed that constituted infection and five that constituted a dry-socket. It is unclear how many of the criteria were required to satisfy the categories. In addition, references to releasing haematomas and placing iodoform drains in some cases makes it difficult to comment, other than to note the higher incidence of dry sockets in cases where partial eruption was previously present.

The cost of removing wisdom teeth in the UK has been calculated to be in the region of $£ 50$ million, although this figure may well have decreased significantly since the publication of guidelines by the National Institute for Clinical Excellence (www.nice.org.uk/ page.aspx?o=509). The bacteriology of the wisdom tooth socket and the research that was related to it was beautifully described by MacGregor. ${ }^{1}$ He concluded, "Antimicrobial drugs appear to have a marginal benefit in third molar surgery when clinically uninfected teeth are removed". Over 20 years later, little appears to have changed!

\section{Practice point}

- There is very little evidence that the routine use of postoperative antibiotics following third molar surgery is indicated.

\author{
Mike Hill \\ Department of Oral Surgery, Medicine and Pathology, Dental \\ School, Wales College of Medicine Cardiff, UK \\ 1. MacGregor AJ. The Impacted Lower Wisdom Tooth, Oxford: Oxford University \\ Press; 1985; p 150.
}

Evidence-Based Dentistry (2005) 6, 10.

doi:10.1038/sj.ebd.6400307 